\title{
Self-reported quality of life scales in women undergoing oocyte freezing versus in vitro fertilization
}

\author{
Sarah S. Lee ${ }^{1}$ (1) $\cdot$ Megan Sutter $^{1} \cdot$ Shelley Lee $^{2} \cdot$ Mindy R. Schiffman $^{2} \cdot$ Yael G. Kramer $^{2} \cdot$ David H. McCulloh $^{2} \cdot$ \\ Frederick Licciardi ${ }^{1,2}$
}

Received: 5 May 2020 / Accepted: 3 August 2020 / Published online: 13 August 2020

(C) Springer Science+Business Media, LLC, part of Springer Nature 2020

\begin{abstract}
Purpose The objective of this study was to investigate stress levels among women undergoing elective oocyte cryopreservation by comparing their self-reported quality of life measures with women undergoing in vitro fertilization during the fertility treatment cycle.

Methods Patients undergoing oocyte retrieval at a single institution were offered a voluntary, anonymous, and written questionnaire. The survey was adapted and validated from the Fertility Quality of Life tool to assess self-reported fertility treatmentrelated problems and was tested for construct validity and reliability. Based on exploratory factor analyses, three subscales were created as follows: fertility treatment-related stress, tolerability, and environment. Relationships between patient characteristics and fertility treatment-related measures were examined with Fisher's exact test, ANOVA, and multivariate regression with significance $p<0.05$.

Results A total of 461 patients (331 IVF, 130 egg freeze) were included in the analysis. Medically indicated egg freezing patients were excluded. Overall, both IVF and egg freeze patients reported stress during the current fertility cycle and there were no significant differences between IVF and egg freeze patients for any subscale scores. Three sets of generalized linear models were run and found age to be associated with fertility treatment-related stress and tolerability scores, with younger patients experiencing greater difficulties. Additionally, patients who underwent repeat cycles reported more fertility treatment-related stress.

Conclusions Patients undergoing egg freezing have similar responses to quality of life questions as patients undergoing IVF. Repeat cycles and younger age contribute to perceptions of stress. This information supports developing stress reduction strategies for all women undergoing egg freezing.
\end{abstract}

Keywords Counseling, $\cdot$ Cryopreservation, $\cdot$ Egg freeze, $\cdot$ Fertility treatment, $\cdot$ Infertility, $\cdot$ Psychology, $\cdot$ Quality of life, $\cdot$ Stress

\section{Introduction}

The link between infertility and stress has been documented throughout the literature. Patients have reported infertility to

Electronic supplementary material The online version of this article (https://doi.org/10.1007/s10815-020-01916-1) contains supplementary material, which is available to authorized users.

Sarah S. Lee

sarah.lee@nyulangone.org

1 Department of Obstetrics and Gynecology, New York University School of Medicine, 550 First Avenue, NBV 9E-2, New York, NY 10016, USA

2 New York University Fertility Center, 660 First Avenue, Fifth floor, New York, NY 10016, USA be one of the most stressful events in their lives [1]. The duration of infertility is correlated with a progression of depressive symptoms, as stress indicators escalate with each cycle as the possibility of treatment failure increases [2, 3]. In addition to the psychological burden imposed by infertility, IVF treatments are physically demanding and taxing of time. They are invasive, requiring frequent office monitoring with venipuncture, transvaginal ultrasounds, weeks of selfinjection with fertility drugs, a procedure under anesthesia for the retrieval of eggs, and the transfer of embryos to the uterus. Moreover, the medications used for the treatments have physical and psychological side effects and may contribute to negative symptoms.

In 2017, of 284,385 assisted reproductive technology (ART) cycles in the USA, 87,535 (31\%) were egg or embryo banking cycles without intent for immediate pregnancy [4]. Egg freezing is now used for oocyte donation, fertility 
preservation in cancer patients, and for elective indications, and is offered by $52-67 \%$ of clinics in the USA [5-7]. Though women undergoing oocyte cryopreservation may face many of the physical and psychological challenges posed by IVF, studies examining the experience of egg freezing or the potential association between egg freezing and psychosocial burdens are understudied. A recent qualitative study of women undergoing elective egg freezing described the egg freezing experience as being influenced by fears of "running out of time," "difficulties in finding a partner," and a "desire to avoid future regrets" [8]. Another survey study among women who previously underwent elective egg freezing found that greater numbers of frozen mature oocytes, increased perceptions of adequate information, and emotional support were associated with less regret [9]. While these studies examined the stress associated with motivation and outcome, we were interested in characterizing the stress associated with the current cycle of ART treatment, (i.e., procedures of stimulation and retrieval). We therefore surveyed women on the day of their egg retrieval about treatment-related stress, and compared the results with those of women undergoing IVF for infertility, a group with previously established and known psychosocial stressors.

\section{Materials and methods}

This was a cross-sectional survey study of all patients undergoing oocyte collection at a single institution from March to August 2017. Patients were initially notified of the voluntary questionnaire during the orientation for fertility treatment. An anonymous written questionnaire was offered to all patients on the day of, but prior to their oocyte retrieval. Inclusion criteria for this study were all women undergoing a cycle of IVF or egg freezing. Of note, patients self-identified as undergoing IVF or egg freezing; those who did not indicate type of fertility treatment were excluded. Patients undergoing egg donation or undergoing egg freezing for medical indications were excluded from the study. The study (S15-02191) and questionnaire were approved by the Institutional Review Board.

\section{Questionnaire design}

The anonymous questionnaire was adapted from the Fertility Quality of Life (FertiQol) tool which assesses the quality of life for patients with infertility, which was developed by an international group of experts. The questions in the original FertiQol evaluate the overall influence of infertility on general health, perceptions, relationships, and the environment and are organized into a "core" section which includes emotional, mind-body, relational, and social subscales, and a "treatment" section which includes the environment and tolerability subscales [10]. Because the original FertiQol assesses the effects of infertility, and because egg freeze patients do not necessarily have the experience of infertility, the original FertiQol could not be used to appropriately measure the stress of women who are electively freezing their oocytes. As there was no validated measure to specifically study the stress and the impact of treatment on two disparate groups, we used items from FertiQol and developed novel items with an expert team of interdisciplinary physicians and psychologists. Some of the questions were revised to reflect the acute changes that occurred as a result of the current cycle in comparison with the overall experience of infertility in the original questionnaire. Questions that did not capture changes in the current cycle were omitted, and eight additional questions that were relevant but not included in FertiQol were added to capture the acute changes that may occur as a result of the current cycle. The additional questions pertained to the tolerability of fertility treatment (e.g., anxiety about injections, side effects). For each question, patients responded to each statement using a 5point Likert scale (e.g., extremely, very much, moderately, a little, not at all). Item responses were scored 0 to 4 with higher scores indicating worse quality of life. See Supplementary Table 1 for all items.

\section{Questionnaire validation}

Two exploratory factor analyses (EFA) using principal axis factoring and varimax rotation were conducted to establish the construct validity of the new questionnaire, first with the Core FertiQol module and new items, then with the Optional FertiQoL module and new treatment-related items based on the following criteria: (1) Eigenvalue $>1.0$, (2) factor loadings $\geq 0.40$ with no cross-loadings $>0.30$ on other factors, (3) and at least three items loading onto a given factor [11]. The first EFA (Core FertiQol) resulted in a one-factor structure with 11 items loading together contrary to the original scale (factor loadings $=0.42-0.80$ ). The items for this factor, fertility treatment-related stress, demonstrated good internal consistency $(\alpha=0.85)$ scored for subsequent analyses. The second EFA (Optional FertiQol module) resulted in a two-factor structure with 7 items loading onto the tolerability subscale (factor loadings $=0.49-0.69$ ) and the original 5 items loading onto the environment subscale (factor loadings $=0.42-0.89$ ). The tolerability and environment subscales demonstrated good internal consistency ( $\alpha \mathrm{s}=0.80$ and 0.85 , respectively). Scoring for the subscales were based on the original FertiQoL to result in a range of scores from 0 to 100 (sum of all items in subscale $\times(25 /$ number of items in subscale $))$. Fertility treatment-related stress scores ranged from 0 to 86 of a possible 100, with higher scores reflecting more stress. Tolerability scores ranged from 0 to 89 and environment scores ranged from 0 to 85 out of a possible 100 with greater scores indicating more difficulty with treatment. For complete results of the EFA analyses, see Supplementary Table 1. 
Table 1 Patient characteristics

\begin{tabular}{|c|c|c|c|c|c|c|c|}
\hline & \multicolumn{7}{|c|}{ Patient type } \\
\hline & \multicolumn{2}{|c|}{ Overall } & \multicolumn{2}{|l|}{ IVF } & \multicolumn{2}{|c|}{ Egg freeze } & \multirow[t]{2}{*}{$p$ Value $^{\mathrm{a}}$} \\
\hline & $n$ & $\%$ & $n$ & $\%$ & $n$ & $\%$ & \\
\hline Overall sample & 461 & $100.0 \%$ & 331 & $71.8 \%$ & 130 & $28.2 \%$ & \\
\hline Age & & & & & & & 0.006 \\
\hline $18-24$ & 4 & $0.9 \%$ & 1 & $0.3 \%$ & 3 & $2.3 \%$ & \\
\hline $25-34$ & 121 & $26.2 \%$ & 85 & $25.7 \%$ & 36 & $27.7 \%$ & \\
\hline $35-44$ & 320 & $69.4 \%$ & 232 & $70.1 \%$ & 88 & $67.7 \%$ & \\
\hline $45-50$ & 12 & $2.6 \%$ & 12 & $3.6 \%$ & 0 & $0.0 \%$ & \\
\hline Missing & 4 & $0.9 \%$ & 1 & $0.3 \%$ & 3 & $2.3 \%$ & \\
\hline Marital status & & & & & & & $<0.001$ \\
\hline Never married & 126 & $27.3 \%$ & 32 & $9.7 \%$ & 94 & $72.3 \%$ & \\
\hline Married/partnered & 296 & $64.2 \%$ & 279 & $84.3 \%$ & 17 & $13.1 \%$ & \\
\hline Divorced/separated & 19 & $4.1 \%$ & 3 & $0.9 \%$ & 16 & $12.3 \%$ & \\
\hline Missing & 20 & $4.3 \%$ & 17 & $5.1 \%$ & 3 & $2.3 \%$ & \\
\hline Highest level of education complete & & & & & & & 0.127 \\
\hline High school & 3 & $0.7 \%$ & 1 & $0.3 \%$ & 2 & $1.5 \%$ & \\
\hline Some college & 17 & $3.7 \%$ & 9 & $2.7 \%$ & 8 & $6.2 \%$ & \\
\hline College & 186 & $40.3 \%$ & 140 & $42.3 \%$ & 46 & $35.4 \%$ & \\
\hline Advanced degree & 252 & $54.7 \%$ & 179 & $54.1 \%$ & 73 & $56.2 \%$ & \\
\hline Missing & 3 & $0.7 \%$ & 2 & $0.6 \%$ & 1 & $0.8 \%$ & \\
\hline Annual household income & & & & & & & $<0.001$ \\
\hline Less than $\$ 49,999$ & 11 & $2.4 \%$ & 6 & $1.8 \%$ & 5 & $3.8 \%$ & \\
\hline$\$ 50,000-\$ 99,999$ & 45 & $9.8 \%$ & 24 & $7.3 \%$ & 21 & $16.2 \%$ & \\
\hline$\$ 100,000-\$ 149,999$ & 84 & $18.2 \%$ & 53 & $16.0 \%$ & 31 & $23.8 \%$ & \\
\hline$\$ 150,000-\$ 199,999$ & 64 & $13.9 \%$ & 43 & $13.0 \%$ & 21 & $16.2 \%$ & \\
\hline Greater than $\$ 200,000$ & 235 & $51.0 \%$ & 186 & $56.2 \%$ & 49 & $37.7 \%$ & \\
\hline Missing & 22 & $4.8 \%$ & 19 & $5.7 \%$ & 3 & $2.3 \%$ & \\
\hline Financing & & & & & & & $<0.001$ \\
\hline None & 5 & $1.1 \%$ & 4 & $1.2 \%$ & 1 & $0.8 \%$ & \\
\hline Public & 1 & $0.2 \%$ & 0 & $0.0 \%$ & 1 & $0.8 \%$ & \\
\hline Private & 232 & $50.3 \%$ & 210 & $63.4 \%$ & 22 & $16.9 \%$ & \\
\hline Self pay & 196 & $42.5 \%$ & 97 & $29.3 \%$ & 99 & $76.2 \%$ & \\
\hline Mixed (private + self pay) & 26 & $5.6 \%$ & 19 & $5.7 \%$ & 7 & $5.4 \%$ & \\
\hline Missing & 1 & $0.2 \%$ & 1 & $0.3 \%$ & 0 & $0.0 \%$ & \\
\hline Repeat cycle & & & & & & & $<0.001$ \\
\hline No & 196 & $42.5 \%$ & 113 & $34.1 \%$ & 83 & $63.8 \%$ & \\
\hline Yes & 243 & $52.7 \%$ & 206 & $62.2 \%$ & 37 & $28.5 \%$ & \\
\hline Missing & 22 & $4.8 \%$ & 12 & $3.6 \%$ & 10 & $7.7 \%$ & \\
\hline
\end{tabular}

${ }^{\text {a }}$ Statistical significance based on Fisher's exact tests, two-tailed, alpha $=0.05$

Additionally, demographic information including age group, education, marital status, mode of payment, and annual household income was collected. Age was grouped in 10-year periods (e.g., 15-24, 25-34, 35-44, 45-54) and was used as a categorical variable. Women were also asked whether the current cycle was the first cycle. Medical indication for fertility treatment was defined as whether patients expected to undergo fertility-reducing treatment (e.g., chemotherapy, radiation, oophorectomy) in the near future. Such patients were excluded from the current study.

\section{Statistical analyses}

Preliminary analyses were conducted to examine patterns of missing data for FertiQol items. Descriptive statistics of patient characteristics and outcome variables (i.e., fertility 
treatment-related problem subscale scores) were computed, as well as normality of outcome variables. Bivariate analyses were conducted among patient characteristics and outcome variables-Fisher's exact tests for categorical variables and one-way analyses of variance (ANOVAs) for continuous variables. Finally, a series of multivariable generalized linear models were used to assess the main effects of patient characteristics and their interactions on quality of life outcome scores. An alpha level of 0.05 was used as the criterion for significance (two-tailed tests). Stata version 15.1 was used to conduct all analyses.

\section{Results}

\section{Overall description of the subjects}

A total of 875 patients underwent oocyte retrieval during the study period. Of these, 232 were egg freeze patients (205 elective, 27 medical indications), and 643 were IVF patients. Four hundred seventy patients responded to the questionnaire, with a response rate of $53.7 \%$. Three patients were excluded due to the incomplete nature of the responses (i.e., did not indicate IVF versus egg freeze), resulting in a total of 467 patients that met our inclusion criteria. Six patients indicated that they were either undergoing fertility preservation prior to a fertility-reducing treatment or were unsure about the possibility of chemotherapy, radiation, or oophorectomy, and these patients all underwent egg freezing. Because of the potential for additional stress and negative responses to treatment that is independent of the fertility factor, these patients were excluded from our analysis, resulting in a final analytic sample of 461. There were 331 IVF patients (71.8\%) and 130 egg freeze patients $(28.2 \%)$ included in the analysis. Little's MCAR test was conducted to determine whether missing data for the FertiQol items were missing at random - the test was not significant $(p=0.740)$, indicating that the data were missing completely at random, and thus, not contributory.

Demographic information is described in Table 1. The majority of patients were between the ages of 35 and 44 for both IVF and egg freeze patients, with significant differences in the distribution of age between the two groups; egg freeze patients tended to represent younger age groups $(p=0.006)$. IVF patients were more likely to be financing the fertility treatment from private insurance, compared with egg freeze patients who were more likely to be paying out of pocket for the fertility treatment $(p<0.001)$. Patients undergoing IVF were more likely to be married or partnered compared with patients undergoing egg freezing, who were less likely to be partnered $(p<0.001)$. Compared with egg freeze patients, IVF patients had higher levels of income, with more annual household incomes greater than $\$ 200,000(p<0.001)$, possibly due to the greater likelihood of IVF patients to be dual-income households. The current cycle was more likely to be a repeat cycle for IVF patients versus egg freeze patients $(62.2 \%$ vs. $28.5 \% ; p<0.001)$.

\section{Comparison of egg freeze patients to IVF patients}

Overall, both IVF and egg freeze patients reported undergoing stress during the current fertility cycle. Three one-way ANOVAs indicated no significant differences between IVF and egg freeze patients on the three subscales (Table 2). Egg freeze patients had similar levels of fertility treatment-related problems, tolerability of the fertility treatment, and had similar satisfaction with the environment as IVF patients.

\section{Demographic associations with outcomes}

To determine if other patient demographics were associated with fertility treatment-related stress, tolerability, and environment scores, a series of ANOVAs were conducted for age (e.g., 13-34 and 35-50), education (e.g., high school/some college; college; and advanced degree), relationship status (e.g., partnered and not partnered), and income (e.g., < $\$ 100,000 ; \$ 100,000$ to $<\$ 150,000 ; \$ 150,000$ to $<$ $\$ 200,000$; and $\$ 200,000+$ ).

There was a significant difference in fertility treatmentrelated stress and tolerability by age $(p s<0.047)$, with patients between ages 18 and 34 reporting more stress $(M=29.34, S D$ $=16.48)$ and problems with tolerating treatment $(M=28.26$, $S D=18.21)$ compared with patients between ages 35 and 50 $(M=25.70, S D=15.30$, and $M=24.54, S D=16.83$, respectively). There was also a significant difference in environment scores by education $(p=0.003)$. Bonferroni-corrected multiple comparisons indicated that those with high school or some college education had a significantly lower average score $(M=$ 9.17, $S D=11.41)$ than those with a college degree $(M=$ $21.42, S D=17.80)$ and advanced degree $(M=23.67, S D=$ 17.49). No other comparisons were significantly different by patient characteristics $(p s>0.05)$.

Table 2 Differences, means, and standard deviations of fertility treatment-related problems subscales by patient type

\begin{tabular}{llll}
\hline \multicolumn{4}{c}{ Patient type } \\
\cline { 2 - 4 } Subscale & IVF & Egg freeze & $p$ Value $^{\mathrm{a}}$ \\
& $M(S D)$ & $M(S D)$ & \\
\hline Fertility treatment-related stress & $26.27(15.17)$ & $27.54(16.92)$ & 0.454 \\
Tolerability & $24.54(16.21)$ & $27.56(19.61)$ & 0.098 \\
Environment & $22.25(17.45)$ & $22.25(18.17)$ & 0.998 \\
\hline a Differences tested using one-way ANOVA tests, two-tailed, alpha $=$ \\
0.05
\end{tabular}




\section{Multivariable analyses}

Three sets of generalized linear models were created with fertility treatment-related stress, tolerability, and environment scores as outcomes and patient type, repeat cycles, and age as independent variables. Women undergoing egg freeze reported similar life stressors as those undergoing IVF. For fertility treatmentrelated stress, patients who completed repeat cycles $(\beta=4.30$, $p=0.009)$ and who were younger $(\beta=-4.60, p=0.009)$ had significantly more stress, but patient type (IVF vs. egg freeze) did not significantly differ $(p=0.189)$. A model with all two-way interactions was computed, but none were significant ( $p \mathrm{~s}>0.05$ ). For tolerability scores, younger patients had significantly greater problems tolerating treatment effects $(\beta=-4.34, p=0.026)$, but no differences emerged for patient type or repeat cycles $(p \mathrm{~s}>$ 0.05). Similarly, no interaction terms were significant when assessed $(p s>0.05)$. For environment scores, patient type, repeat cycles, and age were not significantly related $(p \mathrm{~s}>0.05)$. Controlling for education level did not change any of the aforementioned associations. Figure 1 displays the predicted marginal mean values of the outcome variables by patient characteristics.

\section{Discussion}

This study is the first to show that patients undergoing egg freezing experience as much negative responses and quality of life indicators as patients undergoing IVF. Our multivariable analyses showed that there was no significant difference in the overall quality of life between patients undergoing egg freezing and IVF. Repeat cycle patients were more likely to report treatment-related stress. Women who were older age at first fertility treatment cycle seemed to better tolerate the negative side effects of fertility treatment for both IVF and egg freeze patients.

There are a number of possible reasons why women undergoing egg freezing may experience measurable levels of stress. Although they do not face the immediate concern of embryo development and pregnancy testing, women undergoing egg freezing face anxiety related to the medical process, near term outcome, and ultimate results. In addition, there may be uncertainty of having or later finding a partner to share their lives and help co-parent. Patients may experience some cognitive dissonance relating to starting fertility preservation when they have had long-standing expectations of starting families while young. Grief is a reaction to many forms of loss; unlike the physical loss of a death, which is publicly grieved, the symbolic loss, or the loss of long-held expectations and self-identity that is stripped away by unexpected circumstances, is often a private event endured by patients [12]. In the context of assisted reproductive technologies, the symbolic loss as experienced by some patients may be the loss of the expectation of fertility, natural conception, sexuality, and an easy road to parenting. A patient may appraise and

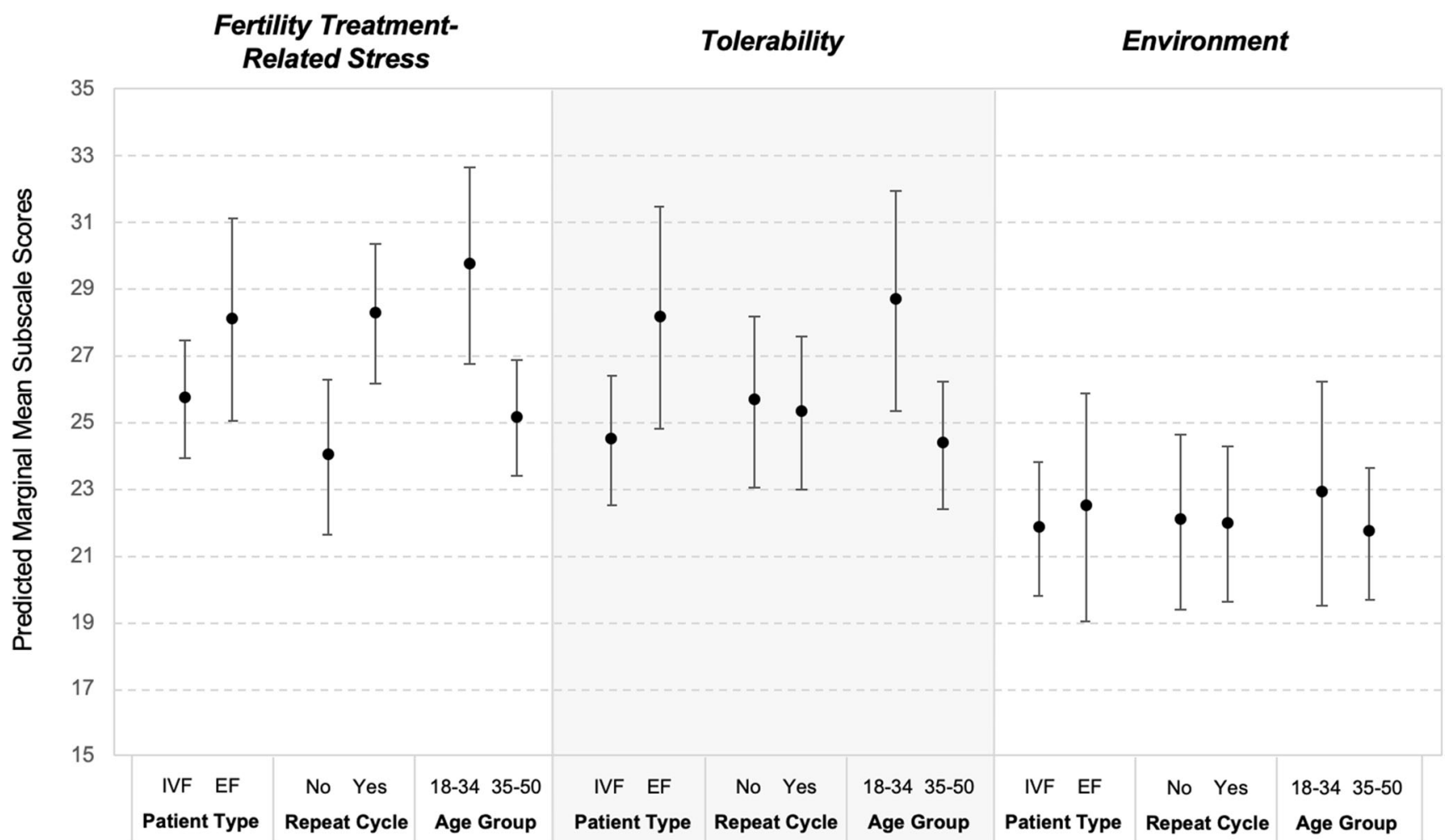

Fig. 1 Predicted marginal means and 95\% confidence intervals of fertility treatment-related stress, tolerability, and environment scores by patient type, repeat cycles, and age group. $\mathrm{IVF}=$ in vitro fertilization. $\mathrm{EF}=$ egg freeze 
react to the start of egg freeze or IVF as a threat to her personal integrity and well-being.

The lack of prior experience with other infertility treatments, the newness of egg freezing as an accepted procedure, and the lack of availability of information from health providers may be related to stress and its effects on mental and physical functioning for egg freeze patients. While the process is gaining increasing public acceptance, to the individual patient, egg freezing as an option to preserve fertility remains a new and an unfamiliar life event. A published survey showed that less than one-third of women undergoing egg freezing reported that they had previously discussed the procedure with the treating gynecologist [13]. Some IVF patients may report lower levels of stress because they may have already had prior experience with infertility treatments before IVF (e.g., natural cycles or intrauterine insemination cycles) or may have spoken to others who have gone through IVF, and thus may have adjusted their expectations and the anticipation of the tolerability of the fertility treatment.

This study highlights the need for offering psychological services to all patients undergoing ART treatment, as counseling can help patients process feelings, navigate stressors, and learn coping strategies as part of the treatment process. Metaanalyses on potential interventions for stress in the infertile population suggest there is a benefit to screening patients for distress at baseline and referring patients for emotional support and coping strategies [14]. In addition, studies suggest that emphasizing education and skills (e.g., cognitive behavioral therapy and mind-body program for infertility) is particularly effective at reducing psychological distress [14-17]. While embracing and incorporating these concepts have been integral to the proper medical and psychological counseling of infertility patients, this study suggests that they should likewise be applied to women undergoing egg freezing.

While this study presents new and important information about the stress of egg freezing, it is not without limitations. Because this was a voluntary and anonymous questionnaire, it is subject to response bias. The survey study was narrow in scope as it focused on the physical and psychosocial experiences of a single cycle; it does not address patients' motivations for pursuing fertility treatment and the effects of the cycle post-treatment. Moreover, we do not know that patients' baseline levels of distress as the survey was collected at a single moment in time. Another major limitation of the study is the time and the method of data collection. The survey was collected in the waiting room for patients undergoing egg retrieval; this procedure along with embryo transfer for IVF patients are particular points of increased stress for patients [15]. If stress had been assessed and compared at various points in the treatment process (e.g., at baseline, after treatment), the results may have varied. On the other hand, patients may underreport symptoms to appear psychologically appropriate for treatment at the time of oocyte retrieval, causing possible biased results. Given that this study design was an anonymous survey in the waiting room, the responses were not able to be correlated with fertility outcomes (e.g., number of oocytes retrieved). Possible future investigation with focus groups and qualitative interviews would be able to capture the nuances in changing attitudes throughout the fertility treatment process for women undergoing egg freeze and IVF. In addition, further studies are needed to investigate the baseline stressors that patients bring to their fertility treatments, and the contributions of preexisting anxiety and stress on the cycle. Additionally, because this study is from a single institution, results may not be generalizable across all populations. Additional studies may elucidate the different responses to fertility treatment by age group for egg freeze patients, as well as possible associations between stress and outcomes of egg freezing.

This is the first study to show that patients undergoing egg freezing experience as much distress during treatment as patients undergoing IVF. This new information can help practitioners appropriately counsel egg freeze patientsboth at the initial consultation and during treatment-on the potential effects of stress on their physical and emotional well-being. Providers should recognize that, as in the case of IVF, women undergoing egg freezing should be made aware that psychological services are readily available, and these patients may benefit greatly from direct referral for counseling.

Authors' contributions All authors on the submission made substantial contribution to the conception or design of work, or the acquisition analysis, or interpretation of data; drafted the work or revised it critically for important intellectual content; approved of the version to be published; and agree to be accountable for all aspects of the work in ensuring that questions related to the accuracy or integrity of any part of the work are appropriately

Data availability Not applicable

\section{Compliance with ethical standards}

Conflict of interest The authors declare that they have no conflict of interest.

Ethical approval All procedures performed in studies involving human participants were in accordance with the ethical standards of the institutional research board (i15-02191) and with the 1964 Helsinki declaration and its later amendments or comparable ethical standards.

Consent to participate Because this was an anonymous survey study, a waiver of informed consent was obtained.

Consent for publication Not applicable

Code availability Not applicable 


\section{References}

1. Klonoff-Cohen H, Chu E, Natarajan L, Sieber W. A prospective study of stress among women undergoing in vitro fertilization or gamete intrafallopian transfer. Fertil Steril. 2001;76(4):675-87.

2. Domar AD, Broome A, Zuttermeister PC, Seibel M, Friedman R. The prevalence and predictability of depression in infertile women. Fertil Steril. 1992;58(6):1158-63.

3. Schaller MA, Griesinger G, Banz-Jansen C. Women show a higher level of anxiety during IVF treatment than men and hold different concerns: a cohort study. Arch Gynecol Obstet. 2016;293(5):113745.

4. Prevention CfDCa. 2017 Assisted Reproductive Technology Fertility Clinic Success Rates Report. United States Department of Health and Human Services: Atlanta; 2019.

5. Cil AP, Seli E. Current trends and progress in clinical applications of oocyte cryopreservation. Curr Opin Obstet Gynecol. 2013;25(3): 247-54.

6. Argyle CE, Harper JC, Davies MC. Oocyte cryopreservation: where are we now? Hum Reprod Update. 2016;22(4):440-9.

7. Lewis EI, Missmer SA, Farland LV, Ginsburg ES. Public support in the United States for elective oocyte cryopreservation. Fertil Steril. 2016;106(5):1183-9.

8. Baldwin K, Culley L, Hudson N, Mitchell H. Running out of time: exploring women's motivations for social egg freezing. J Psychosom Obstet Gynaecol. 2019;40(2):166-73.

9. Greenwood EA, Pasch LA, Hastie J, Cedars MI, Huddleston HG. To freeze or not to freeze: decision regret and satisfaction following elective oocyte cryopreservation. Fertil Steril. 2018;109(6):1097$104 \mathrm{e} 1$.

10. Boivin J, Takefman J, Braverman A. The fertility quality of life (FertiQoL) tool: development and general psychometric properties. Fertil Steril. 2011;96(2):409-15 e3.

11. Osborne JWC, AB KJT. In: Osborne J, editor. Best practices in exploratory factor analysis. Thousand Oaks: Sage Publications; 2008. p. 86-99.

12. Rando TA. Grief, dying, and death : clinical interventions for caregivers, vol. xii. Champaign: Research Press Co; 1984. p. 477.

13. Hodes-Wertz B, Druckenmiller S, Smith M, Noyes N. What do reproductive-age women who undergo oocyte cryopreservation think about the process as a means to preserve fertility? Fertil Steril. 2013;100(5):1343-9.

14. Rich CW, Domar AD. Addressing the emotional barriers to access to reproductive care. Fertil Steril. 2016;105(5):1124-7.

15. Ying L, Wu LH, Loke AY. The effects of psychosocial interventions on the mental health, pregnancy rates, and marital function of infertile couples undergoing in vitro fertilization: a systematic review. J Assist Reprod Genet. 2016;33(6):689-701.

16. Frederiksen Y, Farver-Vestergaard I, Skovgard NG, Ingerslev HJ, Zachariae R. Efficacy of psychosocial interventions for psychological and pregnancy outcomes in infertile women and men: a systematic review and meta-analysis. BMJ Open. 2015;5(1):e006592.

17. Boivin J. A review of psychosocial interventions in infertility. Soc Sci Med. 2003;57(12):2325-41.

Publisher's note Springer Nature remains neutral with regard to jurisdictional claims in published maps and institutional affiliations. 